\title{
New miniaturized Thermopile IR Arrays with medium resolution
}

\author{
M. Schnorr ${ }^{1)}$, B. Forg ${ }^{1)}$, F. Herrmann ${ }^{1)}$, Dr. W. Leneke ${ }^{1)}$, Dr. M. Simon ${ }^{1)}$, \\ Dr. J. Schieferdecker ${ }^{1)}$ \\ ${ }^{1)}$ : Heimann Sensor GmbH, Maria-Reiche-Str. 1, D-01109 Dresden, Tel. 0351-888885-0 \\ info@heimannsensor.com;
}

\begin{abstract}
The recent years showed an increasing demand of low cost and small sized infrared arrays. These arrays can be used for various applications e.g. thermal imaging, industrial process control and person detection. Heimann Sensor improved further the size of the existing arrays so that a $16 \times 16$ and a $32 \times 32$ pixel array fits inside a TO39 housing and the $8 \times 8$ Mini pixel array can be placed inside a TO46 housing. All array types are fully digital and have an integrated EEPROM on which the calibration data is stored.
\end{abstract}

Key words: thermopile array, 16x16, 32×32 and 8x8Mini pixel, TO39 and TO46 housing, infrared detection, fully digital $\left(\mathrm{SPI}, \mathrm{I}^{2} \mathrm{C}\right)$ and calibrated

\section{Introduction}

Heimann Sensor has developed the first fully monolithic thermopile array and the world smallest thermopile sensor in TO housing [1].

These thermal arrays are competitive solutions against other technologies, wherever a thermal picture of the environment is needed and very high resolutions are not necessary, such as person detection, surveillance of temperature critical surfaces, hotspot detection, energy management and security cameras. Other applications can be found in industrial process control and air condition control [2].

The benefits of this technology are low manufacturing costs, low power consumption as well as the avoidance of thermal stabilization or shutters to compensate drift effects.

The new infrared arrays have a decreased size to fit inside a TO39 and TO46 housing. The trend towards small-format infrared arrays is shown in [3].

\section{Decreased size of the 16x16 IR Array}

Until now Heimann Sensor was only able to offer the $16 \times 16$ pixel array in a TO8 housing, due to the new smaller size the array fits inside a TO39 housing now. An EEPROM with a memory size of 2048 byte is placed next to the chip inside the housing. The fully digital interface leads to a 5+1 Pin layout.

\section{Digital interface of the $16 \times 16$ IR Array}

The digital interface requires six pins, two are needed for the voltage supply (3.3V and GND), three for the SPI communication and one for an external clock to drive the sensor. The SPI interface consists of a clock, a Master and Slave select and a bidirectional data channel.

\section{Settings of the 16x16 IR Array}

The sensor has several registers that can be used to change the performance.

The speed of the sensor can be varied from 12.5 to $400 \mathrm{~Hz}$ in factors of 2 via the configuration register.

An IIR and FIR filter can be turned on to reduce the noise.

The amplification and the operating point can be varied to cover a wide temperature range. 


\section{Available optics of the $16 \times 16$ IR Array}

The $16 \times 16$ array can be delivered with several different optics, ranging from $23^{\circ}$ to $90^{\circ} \mathrm{FoV}$.

The different optics are shown in Fig. 1. Thermal screenshots can be seen in Fig. 2 and Fig. 3.

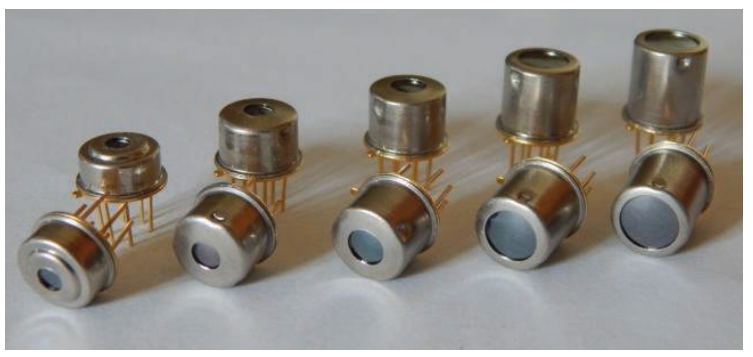

Fig. 1. The different optics that are available for the $16 \times 16$ and $32 \times 32$ pixel array.

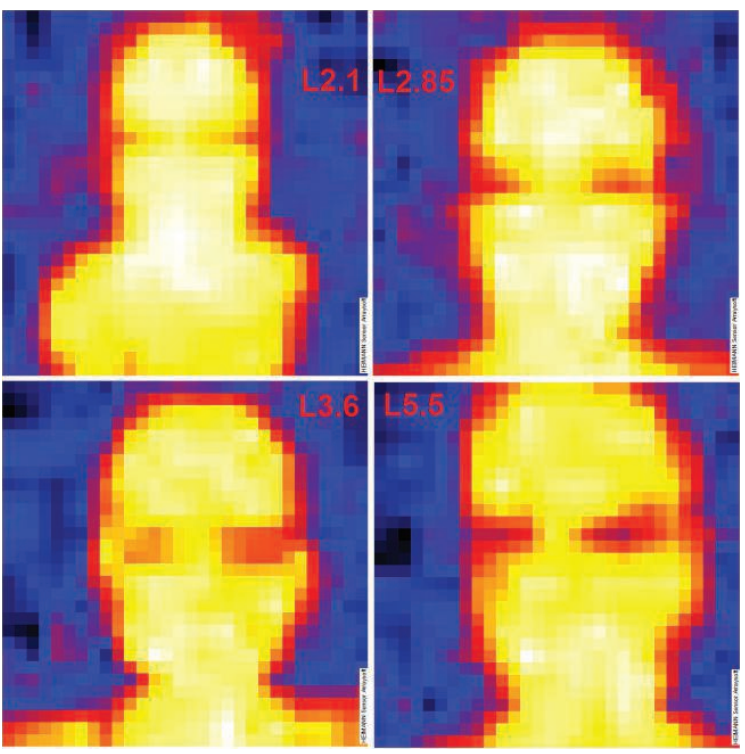

Fig. 3. Picture showing a human head close to the sensor taken with four different optics for the $16 \times 16$ IR Array.

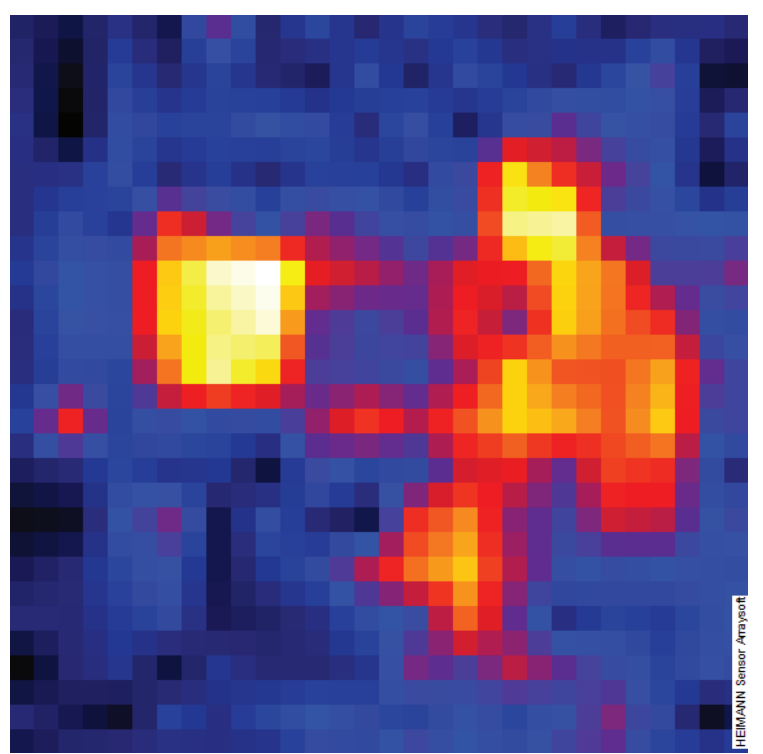

Fig. 2. Picture showing a person sitting at his desk, screenshot captured with the $16 \times 16$ IR array, screenshot taken with the Heimann Sensor ArraySoft. 


\section{Extremely small size of the $8 \times 8$ Mini IR Array}

The new $8 \times 8$ Mini pixel array is the first IR Array that fits inside a TO46 housing. This sensor is having an integrated EEPROM with a memory size of 256 words. Due to the digital $\mathrm{I}^{2} \mathrm{C}$ interface the sensor only needs a $3+1$ Pin layout.

A comparison between the size of this sensor and some competitors is shown in Fig. 4.

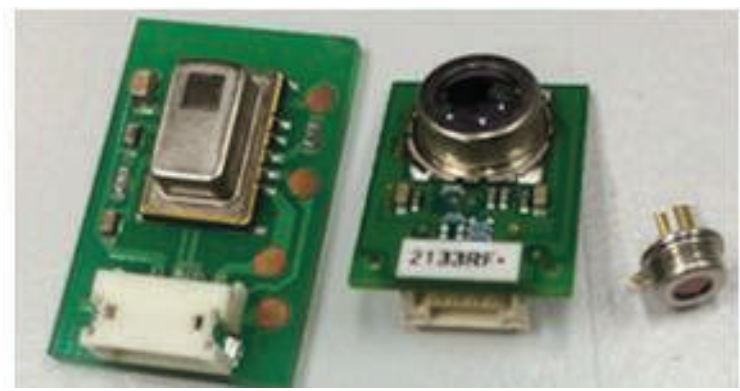

Fig. 4 Picture showing the extremely small size of the sensor, they are from left to right: $8 \times 8$ Array (competitor), $4 \times 4$ array (competitor) and $8 \times 8$ Mini IR Array (Heimann Sensor)

\section{Digital Interface of the 8x8 Mini IR Array}

The digital $I^{2} \mathrm{C}$ interface of the sensor needs 2 pins, the other 2 pins are required for the voltage supply (3.3 $\mathrm{V}$ and GND).

\section{Settings of the $8 \times 8$ Mini IR Array}

The frame rate of the $8 \times 8$ Mini Sensor can be varied from 7 to $88 \mathrm{~Hz}$ in 64 steps.

\section{Available optics of the $8 \times 8$ Mini IR Array}

Currently only one optic was tested with the TO46 housing, this was a L2.1 optic with a FoV of $20^{\circ}$. Heimann Sensor is planning to increase the portfolio of available optics for this type in the future. The sensor can be placed easily inside a TO39 housing and the available optics from the $16 \times 16$ and $32 \times 32$ array can be used.

\section{New 32x32 pixel IR Array}

Heimann Sensor developed a new 32x32 pixel array that is fully digital with an $I^{2} \mathrm{C}$ interface and fitting inside a TO39 housing. Due to the integrated EEPROM inside the cap all calibration data can be stored.

\section{Digital Interface of the $32 \times 32$ IR Array}

The $32 \times 32$ array is designed similar to the $8 \times 8$ Mini array, the $I^{2} \mathrm{C}$ interface requires only 2 pins and the speed can be set up to $1 \mathrm{MHz}$.

\section{Settings of the $32 \times 32$ IR Array}

The sensor is sampling always one quarter of the frame. Which frame shall be sampled can be selected from the outside. The frame rate for a capture of the full frame can be set between 2 and $60 \mathrm{~Hz}$. The frame rate can be varied internally with a clock in 64 steps and by changing the resolution from 16 down to 8 bits in 8 steps.

\section{Available optics of the $32 \times 32$ IR Array}

Due to the same TO39 housing of the $16 \times 16$ pixel array all optics can be used for $32 \times 32$ as well. The pixel pitch of the $32 \times 32$ is roughly half of the $16 \times 16$ which is leading to almost the same resulting field of view of the array.

An overview of the different sensors is shown in Tab. 1 and in Tab. 2 the different field of view is stated.

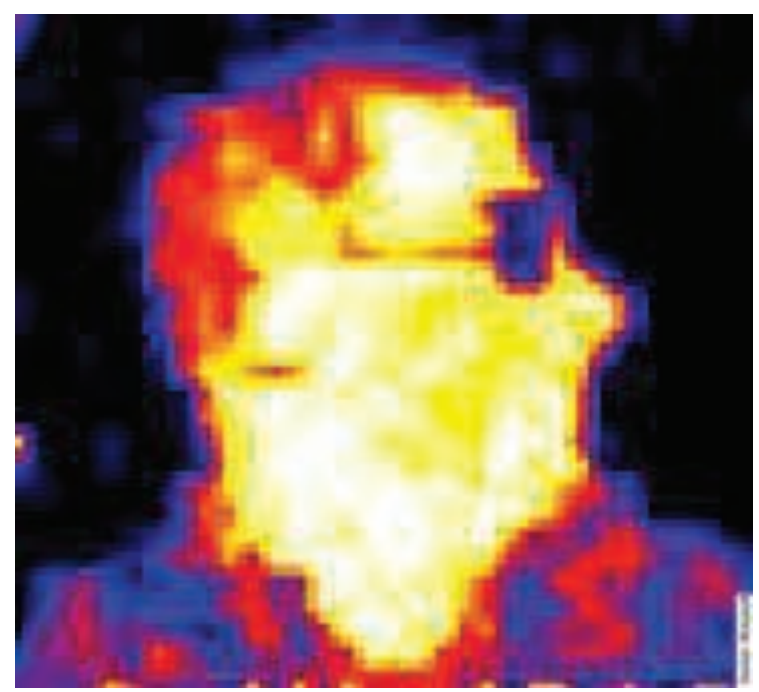

Fig. 5 First IR picture of the new 32x32 Array showing a person looking to the side wearing glasses, captured with the Heimann Sensor ArraySoft. 
Tab. 1: Overview of the different sensors

\begin{tabular}{|l|c|c|c|}
\hline Array & $\mathbf{8 x 8 M i n i}$ & $\mathbf{1 6 \times 1 6}$ & $\mathbf{3 2 \times 3 2}$ \\
\hline Pixelcount & 64 & 256 & 1024 \\
\hline Reference Pixels & 64 & 64 & 64 \\
\hline Pixelsize $[\mu \mathrm{m}]$ & 82 & 150 & 77 \\
\hline Pixel pitch $[\mu \mathrm{m}]$ & 90 & 172.5 & 90 \\
\hline FPS $[\mathrm{Hz}]$ & 7 to 88 & 12.5 to 400 & 2 to 60 \\
\hline FPS steps & 64 & 6 & $64 \times 8$ \\
\hline possible Housing & TO46 \& TO39 & TO39 & TO39 \\
\hline Pin count & 4 & 6 & 4 \\
\hline Interface & $\mathrm{I}^{2} \mathrm{C}$ & SPI & $\mathrm{I}^{2} \mathrm{C}$ \\
\hline FoV $\left[{ }^{\circ}\right]$ & 6 to 20 & 23 to 90 & 23 to 90 \\
\hline NETD $[\mathrm{mK} @ 1 \mathrm{~Hz}]$ & 100 (estimated) & 300 & 150 (estimated) \\
\hline
\end{tabular}

Tab. 2: Overview of the different available optics with their corresponding FoV

\begin{tabular}{|l|c|c|c|c|}
\cline { 3 - 5 } \multicolumn{2}{c|}{} & \multicolumn{3}{c|}{ Field of view [deg] } \\
\hline Optic & Focal Length [mm] & $8 \times 8$ Mini(TO39) & $16 \times 16$ & $32 \times 32$ \\
\hline L2.1 & 2.1 & $20 \times 20$ & $90 \times 90$ & $90 \times 90$ \\
\hline L2.85 & 2.85 & $14 \times 14$ & $52 \times 52$ & $52 \times 52$ \\
\hline L3.6 & 3.6 & $11 \times 11$ & $43 \times 43$ & $43 \times 43$ \\
\hline L5.5 & 5.5 & $7 \times 7$ & $29 \times 29$ & $29 \times 29$ \\
\hline L7.0 & 7.0 & $6 \times 6$ & $23 \times 23$ & $23 \times 23$ \\
\hline
\end{tabular}

\section{References}

[1] W. Leneke, J. Schieferdecker, M. Schulze, M. Simon, K. Storck, B. Forg, F. Völklein:

"Thermopile Linear Array Sensors and Modules with Short Time Constant and High Sensitivity"; Sensor Conference 2007, Proc. Vol.1, Nürnberg, Germany, pp. 329-333

[2] B. Forg, W. Leneke, J. Schieferdecker, M. Schulze, M. Simon, K. Storck: "Thermopile Sensor Arrays with Internal Amplifiers and Digital Out"; Sensor \& Test 2008, Proceedings, Nürnberg, Germany, pp. 249-252.

[3] M. Kimata: "Trends in Small-format Infrared Array Sensors”; Sensors 2013 IEEE, Baltimore, USA, pp. 1-4 